PROCEEDINGS OF THE

AMERICAN MATHEMATICAL SOCIETY

Volume 124, Number 10, October 1996

\title{
CORRESPONDENCES OF CLOSED SUBMODULES
}

\author{
JULIUS M. ZELMANOWITZ
}

(Communicated by Ken Goodearl)

\begin{abstract}
If $N$ is an $M$-faithful $R$-module, then there is an order-preserving correspondence between the closed $R$-submodules of $N$ and the closed $S$ submodules of $\operatorname{Hom}_{R}(M, N)$, where $S=\operatorname{End}_{R} M$.
\end{abstract}

There is a considerable body of research dealing with correspondences between the lattice of submodules of an $R$-module $M$ and the lattice of left ideals of its endomorphism ring $S$. This literature includes the well-known Morita theory and its generalizations. When a complete correspondence between all submodules and all left ideals fails to hold, one may still ask whether there is a correspondence between designated sublattices of submodules and left ideals. One particular situation that has attracted much attention has been cases when there is a correspondence between the closed (i.e., essentially closed) submodules of $M$ and the closed left ideals of $S$. It is known that such a correspondence exists when $M$ is a semisimple module (an elementary observation), when $M$ is a free module [2], when $M$ is a nonsingular retractable module satisfying an additional condition [7], and when $M$ is a nondegenerate module [8]. This article began as a search for a conceptual link among these special cases. Eventually, it was realized that a common denominator is the notion of a self-faithful module, a concept first introduced for generators in [6], and recently exploited to good effect in [3], [4] and [5].

The principal contribution of this article is to demonstrate that a natural correspondence of closed submodules with closed left ideals occurs whenever $M$ is a self-faithful module. In fact, taking a cue from the approach in [1], we show more generally in Theorem 1.2 that when $N$ is an $M$-faithful $R$-module, then there exists an order-preserving correspondence between the closed $R$-submodules of $M$ and the closed $S$-submodules of $\operatorname{Hom}_{R}(M, N)$, where $S=\operatorname{End}_{R} M$. Taking $N=M$ then specializes to the self-faithful case. Additional examples of $M$ being self-faithful, and of the desired correspondence holding, occur when $M$ is a quasi-projective retractable module (Proposition 1.2 in [3]) and when $M$ is a polyform retractable module (Corollary 2.3).

Of purely technical interest is the fact that the results contained in this paper remain true even over rings which fail to have an identity element.

Received by the editors March 7, 1995

1991 Mathematics Subject Classification. Primary 16S50; Secondary 16D70.

(c)1996 American Mathematical Society 


\section{A CORRESPONDENCE OF CLOSED SUBMODULES}

In what follows, $R$ is an associative ring, not necessarily containing an identity element. Unless otherwise indicated, all modules are left modules and homomorphisms are written as right operators. $M$ and $N$ will be left $R$-modules with $U=\operatorname{Hom}_{R}(M, N)$ and $S=\operatorname{End}_{R} M$; $U$ is then a left $S$-module. We write $K \leq L$ to indicate that $K$ is a submodule of $L ; K \leq^{e} L$ means that $K$ is an essential submodule of $L ; K \leq{ }^{c} L$ means that $K$ is an essentially closed submodule of $L$.

For any $R$-module $N$, we set $N_{M}=M \operatorname{Hom}_{R}(M, N)$. $N_{M}$ is the largest submodule of $N$ generated by $M$, and $N$ is called $M$-generated if $N=N_{M}$. We let $\sigma[M]$ denote the class of all submodules of $M$-generated modules. As in [3], a module $N \in \sigma[M]$ is called $M$-faithful if for every $0 \neq g \in \operatorname{Hom}_{R}(X, N)$ with $X \in \sigma[M]$, $\operatorname{Hom}_{R}(M, X) g \neq 0$; equivalently, if for every $0 \neq g \in \operatorname{Hom}_{R}(X, N)$ with $X \leq M^{(t)}$ for some positive integer $t, \operatorname{Hom}_{R}(M, X) g \neq 0$. For instance, when $M$ is $\Sigma$-selfgenerated (that is, when every submodule of every $M^{(t)}$ is $M$-generated), then every $N \in \sigma[M]$ is $M$-faithful and conversely. When $M$ is itself $M$-faithful, $M$ is called a self-faithful module.

Without further ado, we develop the correspondence theorem for closed submodules of an $M$-faithful module.

Proposition 1.1. For $N$ an $M$-faithful $R$-module with $S=\operatorname{End}_{R} M$ and $U=$ $\operatorname{Hom}_{R}(M, N)$, the following conditions hold.

(1) $\operatorname{Hom}_{R}\left(X / X_{M}, N\right)=0$ for every $X \in \sigma[M]$.

(2) If $0 \neq K \leq N$, then $0 \neq K_{M} \leq^{e} K$; in particular, $\operatorname{Hom}_{R}(M, K) \neq 0$.

(3) For every $A \leq{ }_{S} U, A \leq{ }^{e} \operatorname{Hom}_{R}(M, M A)$.

(4) If $K \leq^{e} L \leq N$, then $\operatorname{Hom}_{R}(M, K) \leq{ }^{e} \operatorname{Hom}_{R}(M, L) \leq U$.

(5) If $A \leq^{e} B \leq U$, then $M A \leq^{e} M B \leq N$.

(6) If $K \leq^{c} N$, then $N / K$ is $M$-faithful.

(7) If $K \leq N$, then $K$ is contained in every closed submodule of $N$ which contains $K_{M}$. In particular, if $K \leq^{c} N$, then $K$ is the unique essential closure of $K_{M}$ in $N$.

Condition (1) actually characterizes $M$-faithful modules (see Proposition 1.1 of [3]). The proofs of (2) through (5) appear in [5] for the special case $N=M$, and are repeated here in full generality for the convenience of the reader. (6) and (7) are key steps in securing a correspondence of closed submodules.

Proof. (1) Assume that $X \in \sigma[M]$. If there exists $0 \neq g \in \operatorname{Hom}_{R}\left(X / X_{M}, N\right)$, then $0 \neq \pi g: X \rightarrow N$ where $\pi: X \rightarrow X / X_{M}$ is the natural projection. Since $M_{H o m}(M, X)=X_{M}=\operatorname{ker} \pi, H_{R}(M, X) \pi g=0$, which contradicts the hypothesis that $N$ is $M$-faithful.

(2) If $\iota: K \rightarrow N$ is the natural inclusion map, then $\operatorname{Hom}_{R}(M, K) \iota \neq 0$ because $K \in \sigma[M]$. So $K_{M}=\operatorname{MHom}_{R}(M, K) \neq 0$. If $0 \neq L \leq K$ is given, then $0 \neq L_{M}=$ $M \operatorname{Hom}_{R}(M, L) \subseteq K_{M} \cap L$. Hence $K_{M} \leq^{e} K$.

(3) Let $0 \neq f \in \operatorname{Hom}_{R}(M, M A)$ be given. We may choose a finitely generated submodule $X$ of $M$ and $a_{1}, \ldots, a_{t} \in A$ such that $0 \neq X f \subseteq \operatorname{Hom}_{R}\left(M, \sum_{i=1}^{t} M a_{i}\right)$. Consider the pullback $P=\left\{\left(m, m_{1}, \ldots, m_{t}\right) \in X \times M^{(t)} \mid m f=\sum_{i=1}^{t} m_{i} a_{i}\right\}$ with associated projections $\pi_{X}: P \rightarrow X$ and $\pi: P \rightarrow M^{(t)}$, and let $\varepsilon_{i}: M \rightarrow M^{(t)}$ and $\pi_{i}: M^{(t)} \rightarrow M$ be the canonical injection and projection on the $i$ th coordinate of $M^{(t)}$. We have a commutative diagram 


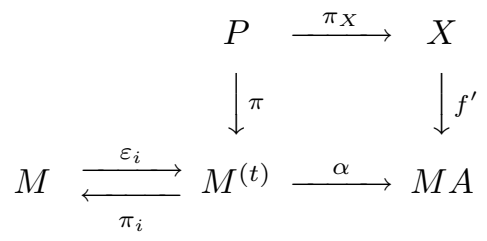

where $\left(m_{1}, \ldots, m_{t}\right) \alpha=\sum_{i=1}^{t} m_{i} a_{i}$ and $f^{\prime}=\left.f\right|_{X}$. Then $0 \neq \pi_{X} f=\pi \alpha: P \rightarrow M A$. Since $P \in \sigma[M]$, there exists $h \in \operatorname{Hom}_{R}(M, P)$ with $h \pi_{X} f \neq 0$. Then we may regard $h \pi_{X}$ as an element of $S$, and so $0 \neq\left(h \pi_{X}\right) f=h \pi \alpha=h \pi\left(\sum_{i=1}^{t} \pi_{i} \varepsilon_{i}\right) \alpha=$ $\sum_{i=1}^{t}\left(h \pi \pi_{i}\right) a_{i} \in A$, proving that $A \leq{ }^{e} \operatorname{Hom}_{R}(M, M A)$.

(4) Suppose that $K \leq \leq^{e} L \leq N$ and let $0 \neq{ }_{S} A \leq \operatorname{Hom}_{R}(M, L)$ be given. Then $0 \neq M A \leq L$, so $M A \cap K \neq 0$. Hence $0 \neq \operatorname{Hom}_{R}(M, M A \cap K) \subseteq$ $H_{R}(M, M A) \cap \operatorname{Hom}_{R}(M, K)$. Since $A \leq \leq^{e} \operatorname{Hom}_{R}(M, M A)$ from (3), it follows that $A \cap \operatorname{Hom}_{R}(M, K) \neq 0$. Thus $\operatorname{Hom}_{R}(M, K) \leq^{e} \operatorname{Hom}_{R}(M, L)$.

(5) Suppose that $A \leq e B \leq U$ and let $0 \neq{ }_{R} L \leq M B$ be given. Then $0 \neq H_{R}(M, L) \leq \operatorname{Hom}_{R}(M, M B)$. Since $A \leq^{e} B \leq^{e} \operatorname{Hom}_{R}(M, M B)$ by (3), $H_{R}(M, L) \cap A \neq 0$. Hence $0 \neq M\left(H_{R}(M, L) \cap A\right) \subseteq L \cap M A$, which proves that $M A \leq \leq^{e} M B$.

(6) Suppose that $0 \neq g \in \operatorname{Hom}_{R}(X, N / K)$ is given with $X \in \sigma[M]$ and $K \leq^{c} N$. By Zorn's lemma, there exists a submodule $L \leq N$ satisfying $K \cap L=0$ and $K \oplus L / K \leq^{e} N / K$. Then $X g \cap(K \oplus L / K) \neq 0$. Set $X^{\prime}=(K \oplus L / K) g^{-1} \leq X$, and let $g^{\prime}$ denote the composition $X^{\prime} \stackrel{\left.g\right|_{X}}{\rightarrow} K \oplus L / K \stackrel{\cong}{\rightrightarrows} L \stackrel{\subseteq}{\rightrightarrows} N$. Then $0 \neq g^{\prime} \in$ $H_{R o m}\left(X^{\prime}, N\right)$ so, by hypothesis, there exists $0 \neq h \in \operatorname{Hom}_{R}\left(M, X^{\prime}\right)$ with $h g^{\prime} \neq 0$. Regarding $h$ as an element of $\operatorname{Hom}_{R}(M, X)$, it follows that $\operatorname{Hom}_{R}(M, X) g \neq 0$, and this proves that $N / K$ is $M$-faithful.

(7) Let $K \leq_{R} N$ and suppose that $K_{M}=\operatorname{MHom}_{R}(M, K) \subseteq L \leq^{c} N$. Then the composition $K \stackrel{\subseteq}{\rightarrow} N \stackrel{\pi}{\rightarrow} N / L$ induces a homomorphism $\iota: K / K_{M} \rightarrow N / L$. From (1) and (6), $\iota=0$, and so $K \subseteq L$.

Theorem 1.2. If $N$ is an $M$-faithful module, then $M A$ has a unique essential closure $(M A)^{c}$ in $N$, for each $A \leq{ }_{S}^{c} U=H_{R} m_{R}(M, N)$, and the assignments

$$
\begin{gathered}
K \longrightarrow \operatorname{Hom}_{R}(M, K) \\
(M A)^{c} \longleftrightarrow A
\end{gathered}
$$

define mutually inverse correspondences between the closed $R$-submodules of $N$ and the closed $S$-submodules of $U=H_{R}(M, N)$.

Proof. If $K \leq^{c} N$ and $\operatorname{Hom}_{R}(M, K) \leq^{e} A \leq{ }_{S} U$ then, from (5), $\operatorname{MHom}_{R}(M, K)$ $\leq^{e} M A$. Applying (7), we learn that $M A \leq^{e} K$. Hence $A \subseteq H_{R}(M, K)$, so $A=H_{R}(M, K)$, proving that $H_{R}(M, K) \leq{ }^{c} U$. Also, from (7), $K=K_{M}{ }^{c}=$ $\left(M \operatorname{Hom}_{R}(M, K)\right)^{c}$.

Suppose that $A \leq{ }_{S}{ }_{S} U$; we first show that $M A$ has a unique closure in $N$. Say $M A \leq^{e} K \leq^{c} N$. Then, from (3) and (4), $A \leq^{e} \operatorname{Hom}_{R}(M, M A) \leq{ }^{e} \operatorname{Hom}_{R}(M, K)$, from which it follows that $A=\operatorname{Hom}_{R}(M, K)$. Therefore, $M A=M \operatorname{Hom}_{R}(M, K)$ $=K_{M}$, which has $K$ as its unique essential closure, by (7). Thus $A=\operatorname{Hom}_{R}(M, K)$ $=H_{R}\left(M,(M A)^{c}\right)$, and with this, the proof is completed.

A $C S$-module is one in which every closed submodule is a direct summand; a left $C S$-ring is a ring which is a $C S$-module over itself. 
Corollary 1.3. For $N$ an $M$-faithful module, the following are true.

(1) ${ }_{R} N$ and ${ }_{S} U$ have the same uniform dimension; i.e., an internal direct sum of submodules of one of these modules gives rise to an internal direct sum in the other module with the same cardinal number of summands.

(2) $(M A)^{c}=M A$ for each $A \leq^{c}{ }_{S} U$ if and only if each $K \leq^{c} N$ is $M$-generated.

(3) If ${ }_{R} N$ is a CS-module, then ${ }_{S} U$ is a CS-module; and the converse holds when $N$ is $M$-generated.

Proof. The proof of (1) is straightforward and is left to the reader.

(2) Suppose that $(M A)^{c}=M A$ for each $A \leq{ }^{c}{ }_{S} U$ and let $K \leq{ }^{c} N$ be given. Then $\operatorname{Hom}_{R}(M, K) \leq^{c} U$ so, from the theorem and the hypothesis, $K=$ $\left(M_{H o m}(M, K)\right)^{c}=M \operatorname{Hom}_{R}(M, K)$. Thus, $K$ is $M$-generated.

Conversely, suppose that each $K \leq^{c} N$ is $M$-generated and let $A \leq^{c}{ }_{S} U$ be given. From the theorem, $A=\operatorname{Hom}_{R}\left(M,(M A)^{c}\right)$. On the other hand, $(M A)^{c}=$ $M_{H o m}\left(M,(M A)^{c}\right)$ by hypothesis, so $(M A)^{c}=M A$.

For (3), suppose that ${ }_{R} N$ is a $C S$-module and that $A \leq^{c} U$. Then $N=$ $(M A)^{c} \oplus L$ for some $L \leq N$. Hence $U=\operatorname{Hom}_{R}(M, N)=\operatorname{Hom}_{R}\left(M,(M A)^{c}\right) \oplus$ $\operatorname{Hom}_{R}(M, L)=A \oplus \operatorname{Hom}_{R}(M, L)$.

For the converse, assume that $N$ is $M$-generated and let ${ }_{S} U$ be a $C S$-module and $K \leq{ }^{c} N$. Then $U=\operatorname{Hom}_{R}(M, K) \oplus B$ for some $B \leq{ }_{S} U$. Hence $N=$ $M_{H o m}(M, N)=M H o m_{R}(M, K)+M B=K+M B$. This last sum is direct because $K \cap M B \neq 0$ would imply that $0 \neq \operatorname{Hom}_{R}(M, K \cap M B) \subseteq \operatorname{Hom}_{R}(M, K) \cap$ $\operatorname{Hom}_{R}(M, M B)$, whence $0 \neq \operatorname{Hom}_{R}(M, K) \cap B$, a contradiction. So $N=K \oplus M B$, and the proof is completed.

\section{Applications to Retractable modules}

Observe that an $M$-faithful module $N$ is $M$-retractable in the sense that $\operatorname{Hom}_{R}(M, X) \neq 0$ for every $0 \neq X \leq N$ (Proposition 1.1(2)). When $M$ is itself $M$-retractable, we say simply that $M$ is retractable. Our next objective is to show that $M$-retractability characterizes $M$-faithfulness for some important families of modules.

An $R$-module $M$ is called polyform if every homomorphism $f \in \operatorname{Hom}_{R}(X, M)$ with $X \leq M$ has a closed kernel in $X$. Such a homomorphism is called a partial homomorphism from $M$ into itself. Nonsingular modules form a proper subclass of polyform modules, as do semisimple modules. We begin by showing that when $M$ is polyform, so are many modules in $\sigma[M]$.

Lemma 2.1. Suppose that $\left\{M_{i} \mid i \in I\right\}$ is a family of modules such that partial homomorphisms from $M_{i}$ to $M_{j}$ have closed kernels for each $i, j \in I$, and let $K_{i} \leq^{c}$ $M_{i}$ for each $i \in I$. Then $\bigoplus_{i \in I} M_{i} / K_{i}$ is a polyform module.

Proof. From Proposition 3.3 of [9], it suffices to show that a nonzero partial homomorphism from $M_{1} / K_{1}$ to $M_{2} / K_{2}$ does not have an essential kernel. Let $0 \neq$ $f \in \operatorname{Hom}_{R}\left(N_{1} / K_{1}, M_{2} / K_{2}\right)$ be given with $K_{1} \leq N_{1} \leq M_{1}$. As in the proof of Proposition 1.1(6), one may choose $K_{1} \leq L_{1} \leq N_{1}$ and $0 \neq L_{2} \leq M_{2}$ such that $K_{2} \cap L_{2}=0$ and $0 \neq\left(L_{1} / K_{1}\right) f \subseteq K_{2} \oplus L_{2} / K_{2}$. Set $f^{\prime}=\left.f\right|_{L_{1} / K_{1}}$ and let $\pi: L_{1} \rightarrow L_{1} / K_{1}$ denote the quotient homomorphism. By hypothesis, the composition $L_{1} \stackrel{\pi}{\rightarrow} L_{1} / K_{1} \stackrel{f^{\prime}}{\rightarrow} K_{2} \oplus L_{2} / K_{2} \stackrel{\cong}{\rightrightarrows} L_{2} \stackrel{\subseteq}{\rightrightarrows} M_{2}$ must have a closed kernel. From this it follows that the kernel of $f$ is not essential in $N_{1} / K_{1}$. 
Proposition 2.2. (1) Suppose that $M$ is a quasi-projective module and that $N \in$ $\sigma[M]$. Then $N$ is $M$-faithful if and only if $N$ is $M$-retractable.

(2) Suppose that $M$ is a polyform module and that $N \leq M^{(I)} / K$ for some index set $I$ and $K \leq{ }^{c} M^{(I)}$. Then $N$ is $M$-faithful if and only if $N$ is $M$-retractable.

(3) Suppose that $\left(R, M, M^{\prime}, R^{\prime}\right)$ is a Morita context and that $N \in \sigma[M]$ is an $R$-module which satisfies $\left(M, M^{\prime}\right) n \neq 0$ for any $0 \neq n \in N$. Then $N$ is $M$-faithful.

Proof. The proof of (1) is straightforward and is given in Proposition 1.2 of [3].

(2) Suppose that $N \leq M^{(I)} / K$ and that $N$ is $M$-retractable. It suffices to prove that $\operatorname{Hom}_{R}(M, X) g \neq 0$ whenever $0 \neq X \leq M^{(t)}$ for some integer $t$ and $0 \neq g \in$ $\operatorname{Hom}_{R}(X, N)$. We may regard $g$ as a partial endomorphism of $M^{(t)} \oplus\left(M^{(I)} / K\right)$, which is a polyform module by the preceding lemma. Hence there exists $0 \neq X^{\prime} \leq X$ such that $\left.g\right|_{X^{\prime}}$ is a monomorphism, and thus $X^{\prime}$ is isomorphic to a submodule of $N$. By hypothesis, there exists $0 \neq h \in \operatorname{Hom}_{R}\left(M, X^{\prime}\right) \subseteq \operatorname{Hom}_{R}(M, X)$. Then $h g \neq 0$, completing the proof.

(3) As in (2), it suffices to prove that $\operatorname{Hom}_{R}(M, X) g \neq 0$ whenever $0 \neq X \leq M^{(t)}$ for some integer $t$ and $0 \neq g \in \operatorname{Hom}_{R}(X, N)$. Choose any $x \in X$ with $0 \neq x g \in N$. By hypothesis, there exist $m \in M$ and $m^{\prime} \in M^{\prime}$ with $\left(m, m^{\prime}\right)(x g) \neq 0$. Define $h \in \operatorname{Hom}_{R}(M, X)$ by $n h=\left(n, m^{\prime}\right) x$ for any $n \in M$. Then $h g \neq 0$ because

$$
m h g=\left(\left(m, m^{\prime}\right) x\right) g=\left(m, m^{\prime}\right)(x g) \neq 0 .
$$

We now combine the information in the previous proposition with that in Theorem 1.2 and Corollary 1.3 in the special case when $N=M$ and $U=S$.

Corollary 2.3. Suppose that either (i) $M$ is a quasi-projective retractable module, or (ii) $M$ is a polyform retractable module, or (iii) there exists a Morita context $\left(R, M, M^{\prime}, R^{\prime}\right)$ such that $\left(M, M^{\prime}\right) m \neq 0$ for any $0 \neq m \in M$, or (iv) $M$ is a $\Sigma$-self-generator.

Then the assignments in Theorem 1.2 define mutually inverse lattice correspondences between the closed submodules of $M$ and the closed left ideals of $S=$ $\operatorname{End}_{R} M ; M$ is a CS-module if and only if $S$ is a left $C S$-ring; and the uniform dimension of $M$ equals the left uniform dimension of $S$.

The instances (i)-(iv) in the preceding corollary include and generalize a number of previously studied situations. The correspondence of closed submodules with closed left ideals had been known in the following cases.

- Semisimple modules - these are covered by (i) and (iv).

- Free modules over a ring with identity element, [2] — these are covered by (i) and (iii).

- Nonsingular retractable modules which satisfy the condition given in Proposition 1.1(3), [7] - these are a special case of (ii), so that, for a nonsingular module, retractability is enough for the correspondence reported in [7] to hold, without additional conditions.

- Nondegenerate modules, [8] — these are included in (iii).

Final comments. The fact that $M$ is a $C S$-module if and only if $S$ is a left $C S$-ring when $M$ is self-faithful was first shown in [4]. For semisimple or free modules, Corollary 1.3(2) applies, so that $M A \leq^{c} M$ for each $A \leq^{c} S$ in these cases. 


\section{REFERENCES}

[1] T. Albu and C. Nastasescu, Relative finiteness in module theory, Marcel Dekker, New York, 1984. MR 85k: 16001

[2] A. W. Chatters and S. M. Khuri, Endomorphism rings of modules over non-singular CSrings, J. London Math. Soc. (2) 21 (1980), 434-444. MR 82m:16033

[3] J. L. García Hernández and J. L. Gómez Pardo, Self-injective and PF endomorphism rings, Israel J. Math. 58 (1987), 324-350. MR 89b:16041

[4] J. L. García and M. Saorín, Endomorphism rings and category equivalences, J. Algebra 127 (1989), 182-205. MR 91b:16009

[5] J. L. Gómez Pardo, Endomorphism rings and dual modules, J. Algebra 130 (1990), 477-493. MR 91f: 16040

[6] T. Kato, U-distinguished modules, J. Algebra 25 (1973), 15-24. MR 49:5100

[7] S. M. Khuri, Nonsingular retractable modules and their endomorphism rings, Bull. Austral. Math. Soc. 43 (1991), 63-71. MR 92b:16064

[8] Zhou Zhengping, Correspondence theorems for nondegenerate modules and their endomorphism rings, Proc. Amer. Math. Soc. 121 (1994), 25-32. MR 94g:16040

[9] J. M. Zelmanowitz, Representation of rings with faithful polyform modules, Comm. Algebra 14 (1986), 1141-1169. MR 87e:16078

Department of Mathematics, University of California, Santa Barbara, California 93106

E-mail address: julius@math.ucsb.edu 